\title{
Strain Mapping with Electron Back Scatter Diffraction: Sensitivity Studies and Pattern Remapping
}

\author{
Jun Jiang $^{1}$, T. Ben Britton ${ }^{2}$, Angus J. Wilkinson ${ }^{1}$ \\ 1. Department of Materials, University of Oxford, Parks Road, Oxford OX1 3PH, UK. \\ 2. Department of Materials, Imperial College London, Exhibition Road, London, SW7 2AZ, UK.
}

The implementation of cross-correlation analysis of EBSD patterns has allowed measurements with much improved angular sensitivity including the ability to determine the variations in elastic strains and stresses. Cross-correlation is used to measure shifts between sub-regions of test and reference patterns and a simple geometric analysis then allows the elastic strains and lattice rotations to be calculated from the measured dispersion of pattern shifts. The original paper reported the pattern shifts were measured to a precision of $\sim \pm 0.05$ pixels corresponding to strain and rotation sensitivity of $\sim \pm 10^{-4}[1]$.

The first part of this paper reports a strain sensitivity study of how the pattern shift sensitivity is affected by data collection, particularly exposure times and camera binning. Patterns were recorded using an 11 point line scan along the tilt axis (x-axis) of a $\mathrm{Si} 001$ sample with a $10 \mu \mathrm{m}$ step size. This was undertaken at camera binning levels of $1 \times 1,2 \times 2,4 \times 4$ and $8 \times 8$ for which exposure times of $1.04 \mathrm{~s}, 0.24 \mathrm{~s}$, $0.05 \mathrm{~s}$ and $0.02 \mathrm{~s}$ were used. Each line scan was repeated 5 times to provide multiple exposures from similar locations that could then be averaged so as to improve the signal to noise ratio. Each line scan was treated as 10 pattern pairs with identical $10 \mu \mathrm{m}$ steps between them. The standard deviation in $\mathrm{x}$ and y pattern shifts were calculated for these repeat measurements and these were then averaged over any repeat line scans available to give a single parameter we report as the pattern shift precision. In each case we report this pattern shift precision as the Euclidian shift in terms of fractions of a raw pixel in the unbinned patterns. Results from this analysis are presented in figure 1. For a single EBSD exposure using different hardware binning (fig la) the highest precision, \pm 0.04 pixels, was obtained using no binning (1x1), and as expected the precision worsens significantly as the binning is increased, to \pm 0.73 pixels with $8 \times 8$ binning. Software binning can be used to generate patterns at different binning levels but similar exposure times. The five line scans at $1 \mathrm{x} 1$ binning were software binned and figure $1 \mathrm{~b}$ shows at $1 \times 1$ and $2 \times 2$ binning similar precision are obtained of \pm 0.018 and \pm 0.019 pixels respectively, while with greater binning the precision deteriorates. Notably even for $8 \times 8$ binning the precision is better than \pm 0.05 pixels. The similarity of the $1 \times 1$ and $2 \times 2$ binning results indicates that there is in fact little real gain in information content afforded by the additional pixels. Finally in figure $1 \mathrm{c}$ the detector binning is fixed at the highest resolution 1x1 binning and the effect of software integration over increasing total exposure times is shown. The precision improves with increasing exposure times but the response is non-linear with an ultimate precision of \pm 0.019 pixels with five seconds exposure. Sensitivity gains beyond this would require drastic increases in total exposure time and would not realistic for mapping operations.

The second part of this paper concerns analysis of deformed metals in which significant lattice rotations develop as a result of the stored dislocation content. The pattern shifts from the rotations can be much larger than for those due to elastic strains and therefore the mapping between sub-regions on test and reference pattern is not so well represented by a simple linear translation. This results in significant errors being induced in the lattice strain variation [2]. To overcome this problem a 'remapping algorithm' has been developed [3]. An initial pass of cross-correlation analysis is used to estimate the 
rigid body rotation between the test and reference lattice. This rotation is then used to remap the pattern intensity within the test pattern so as to bring it in closer (crystal) alignment with the reference pattern. A second pass of cross-correlation analysis is then undertaken and the elastic strains and a small correction to the rotation are determined. These are all combined to report variations in (finite) lattice rotation and the Green's strain tensor. Figure 2 illustrates the effect of applying the remapping algorithm. The EBSD map was obtained over a $112 \mu \mathrm{m}$ square array with step size $0.5 \mu \mathrm{m}$ from a $\mathrm{Cu}$ polycrystal deformed in uniaxial tension to $10 \%$ strain. Grains were defined using a $7^{\circ}$ threshold and reference patterns automatically assigned for each grain. Each pass of cross-correlation analysis used 100 sub-regions and in a post processing step the average elastic strain in each grain was brought to zero. Figure 2 shows the type III residual elastic strain variations transverse to the tensile axis; (a) as measured with the first pass of cross-correlation and (b) after remapping and a second pass of crosscorrelation analysis. It is obvious that the remapping significantly reduces the magnitude of the strain variations with large regions of high strains shown in fig $2 a$, moving to more moderate values in fig $2 b$. Figure 2c shows a histogram of the strain levels present in fig $2 \mathrm{a}$ and $\mathrm{b}$ which again shows that the remapping reduces the high strain magnitude tails present in the distribution and strengthens the central low strain peak.

[1] A.J. Wilkinson, G. Meaden and D.J. Dingley, Ultramicroscopy, 106 (2006) 307-313.

[2] T.B. Britton and A.J. Wilkinson, Ultramicroscopy, 111 (2011) 1395-1404.

[3] T.B. Britton and A.J. Wilkinson, Ultramicroscopy, 114 (2012) 82-95.
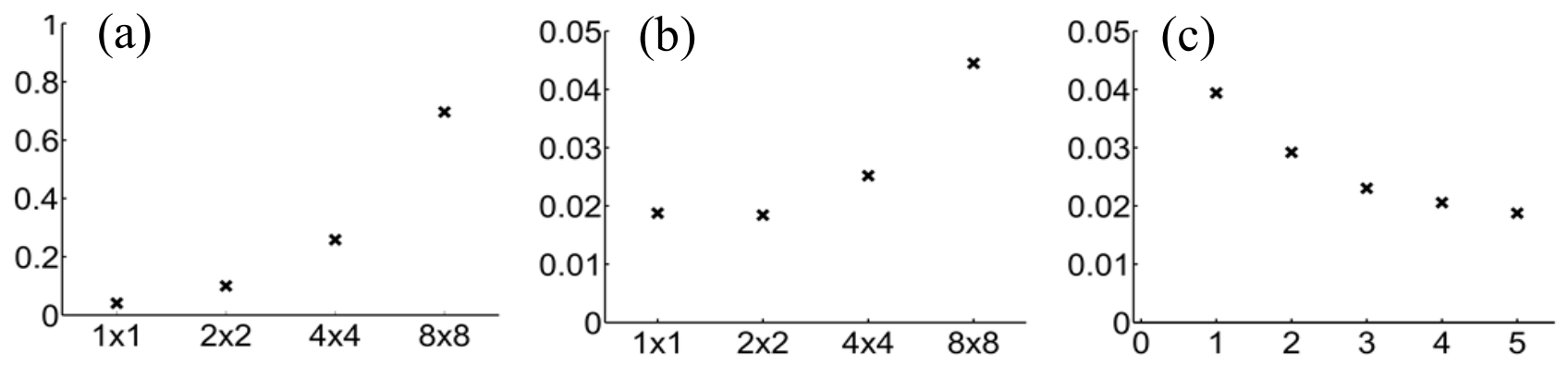

Figure 1. Pattern shift precision in raw unbinned pixels as a function of: (a) on camera binning (varying exposure time), (b) software binning (fixed exposure time), (c) exposure time (fixed 1x1 binning).
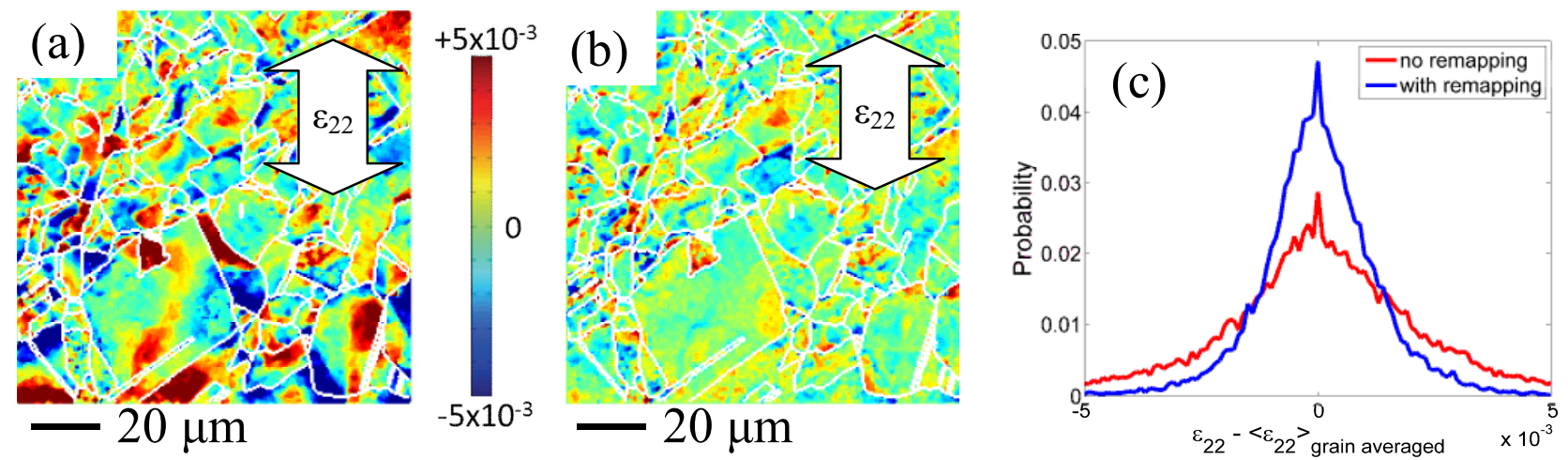

Figure 2. Transverse strains in $\mathrm{Cu}$ polycrystal after 10\% tensile strain. (a) no remapping, (b) with remapping, (c) histogram of strain distributions with and without remapping. 